\title{
BMJ Open Endovascular repair or open repair for ruptured abdominal aortic aneurysm: a Cochrane systematic review
}

\author{
S A Badger, ${ }^{1}$ D W Harkin, ${ }^{2}$ P H Blair, ${ }^{2}$ P K Ellis, ${ }^{2}$ F Kee, ${ }^{3}$ R Forster ${ }^{4}$
}

To cite: Badger SA, Harkin DW, Blair PH, et al. Endovascular repair or open repair for ruptured abdominal aortic aneurysm: a Cochrane systematic review. BMJ Open 2016;6:e008391.

doi:10.1136/bmjopen-2015008391

- Prepublication history for this paper is available online. To view these files please visit the journal online (http://dx.doi.org/10.1136/ bmjopen-2015-008391).

Received 10 April 2015 Revised 19 August 2015 Accepted 21 August 2015

CrossMark

\begin{abstract}
${ }^{1}$ Mater Misericordiae University Hospital, Dublin, Ireland

${ }^{2}$ Belfast Vascular Centre, Royal Victoria Hospital, Belfast, UK

${ }^{3}$ Centre for Public Health, Queens University Belfast, Belfast, UK

${ }^{4}$ Centre for Population Health

Sciences, University of

Edinburgh, Edinburgh, UK
\end{abstract}

Correspondence to

DW Harkin;

denis.harkin@belfasttrust.

hscni.net

\section{ABSTRACT}

Objectives: Emergency endovascular aneurysm repair (eEVAR) may improve outcomes for patients with ruptured abdominal aortic aneurysm (RAAA). The study aim was to compare the outcomes for eEVAR with conventional open surgical repair for the treatment of RAAA.

Setting: A systematic review of relevant publications was performed. Randomised controlled trials (RCTs) comparing eEVAR with open surgical repair for RAAA were included.

Participants: 3 RCTs were included, with a total of 761 patients with RAAA.

Interventions: Meta-analysis was performed with fixed-effects models with ORs and $95 \% \mathrm{Cls}$ for dichotomous data and mean differences with $95 \% \mathrm{Cls}$ for continuous data.

\section{Primary and secondary outcome measures:}

Primary outcome was short-term mortality. Secondary outcome measures included aneurysm-specific and general complication rates, quality of life and economic analysis.

Results: Overall risk of bias was low. There was no difference between the 2 interventions on 30-day (or in-hospital) mortality, OR 0.91 (95\% Cl 0.67 to 1.22 ; $\mathrm{p}=0.52$ ). 30-day complications included myocardial infarction, stroke, composite cardiac complications, renal complications, severe bowel ischaemia, spinal cord ischaemia, reoperation, amputation and respiratory failure. Reporting was incomplete, and no robust conclusion was drawn. For complication outcomes that did include at least 2 studies in the meta-analysis, there was no clear evidence to support a difference between eEVAR and open repair. Longer term outcomes and cost per patient were evaluated in only a single study, thus precluding definite conclusions.

Conclusions: Outcomes between eEVAR and open repair, specifically 30-day mortality, are similar. However, further high-quality trials are required, as the paucity of data currently limits the conclusions.

\section{BACKGROUND}

Abdominal aortic aneurysm (AAA) affects between $1.2 \%$ and $7.6 \%$ of the population over 50 years of age in the UK. There has

\section{Strengths and limitations of this study}

- This is the first study to do a meta-analysis of the three major randomised controlled trials comparing endovascular and open repair of abdominal aortic aneurysms.

- The major finding is that there is no difference seen between operative modalities for ruptured aneurysm repair in terms of morbidity or mortality.

- The quality of the study is reduced by the lack of complete reporting in the trials.

- Long-term outcome data would enhance the results of the meta-analysis and is still awaited.

- Robust conclusions are difficult to draw due to paucity of data, but one modality should not be favoured over another based on current research.

been recent decline in prevalence to about $2 \%$. This is independent of participant selection criteria and reflects better cardiovascular risk profiling and management in the overall population. ${ }^{1}{ }^{2}$ Unfortunately, many aneurysms first present as a rupture resulting in death in the majority of those affected.

The high mortality rate from ruptured AAA (RAAA) is well recognised. Clinicians have been reticent to apply scoring systems rigidly as to do so would condemn many to certain death. $^{3-5}$ Patients who undergo successful open repair of RAAA enjoy a postoperative quality of life similar to the "normal population'. ${ }^{6}$ The risk of aneurysm rupture is proportional to aneurysm size, with aneurysms measuring less than $5.4 \mathrm{~cm}$ having an annual rupture rate of approximately $1 \%$ whereas those greater than $7.0 \mathrm{~cm}$ in diameter have an annual rupture rate of $32.5 \% .^{89}$ Currently, rupture leads to death in over $80 \%$ of those affected, including 30-65\% of those who receive conventional open surgical repair and is responsible for $2.1 \%$ of all deaths in men over 65 years. ${ }^{8}{ }^{10-12}$ These findings contrast with the significantly better outcome if conventional open surgical repair of the AAA is planned before rupture can occur. 
Historically, open surgical repair was the only effective treatment for AAA. This major operation carries significant morbidity and mortality, due to the combined effects of surgical exposure, haemorrhage and aortic clamping with related lower torso ischaemia-reperfusion injury. However, with improved patient selection and perioperative care, some specialist centres are reporting mortality rates of less than $2 \%$ and surgeons in nonspecialist units achieving mortality rates of $5-8 \% .^{8-10}$ However, in the past two decades, this treatment has been challenged by endovascular aneurysm repair (EVAR). Significant advancements in commercial stent design, delivery and implantation technique have made this a valuable alternative to open repair in selected suitable cases. ${ }^{13-15}$ Its use in elective cases has reduced early postoperative morbidity and mortality. ${ }^{16}{ }^{17}$ Its minimally invasive nature allows it to be performed under regional or even local anaesthesia, rather than general anaesthesia. This allows repair in patients with significant concomitant medical disease who may otherwise have been considered unfit for surgery. ${ }^{10}{ }^{18}$ Two recent large prospective randomised controlled trials (RCTs) have shown reduced early complications and mortality with EVAR. ${ }^{16}{ }^{17}$ However, while elective endovascular repair clearly has a role, these trials reinforce the knowledge

Figure 1 PRISMA flow chart of literature selection. TSC, Trials Search Co-ordinator. that open repair will remain the treatment for a large proportion of patients whose AAA is unsuitable on anatomical grounds for EVAR. ${ }^{16} 19{ }^{20}$ In addition, long-term results from the EVAR 1 trial should add caution, with later ruptures in the EVAR group, and no long-term difference in the all-cause mortality. ${ }^{21}$

Rupture of an AAA (RAAA) is a catastrophic event, with up to $80 \%$ mortality. ${ }^{22}{ }^{23}$ RAAA exposes the patients to the combined injury of a period of haemorrhagic shock and lower torso ischaemia followed by a reperfusion injury on successful revascularisation. This 'two hit' mechanism of injury initiates a systemic inflammatory response syndrome, leading to a multiple organ dysfunction syndrome (MODS). ${ }^{24}$ MODS is the primary cause of $70 \%$ of such deaths and a contributory cause of the remainder. ${ }^{25} 26$ The multiorgan failure, despite intensive care support, once established leads to death in over $70 \%$ of cases. ${ }^{25}{ }^{27}$ EVAR, especially under local anaesthesia, reduces the physiological insult to the body as compared with conventional open surgical repair. ${ }^{28} 29$ Emergency EVAR (eEVAR) appears to offer a feasible alternative to conventional open repair in selected patients. ${ }^{29}{ }^{30}$ Emergency cases were repaired with an aortouniiliac stent graft, contralateral occluder and fem-fem crossover in the initial years, due to increased speed of

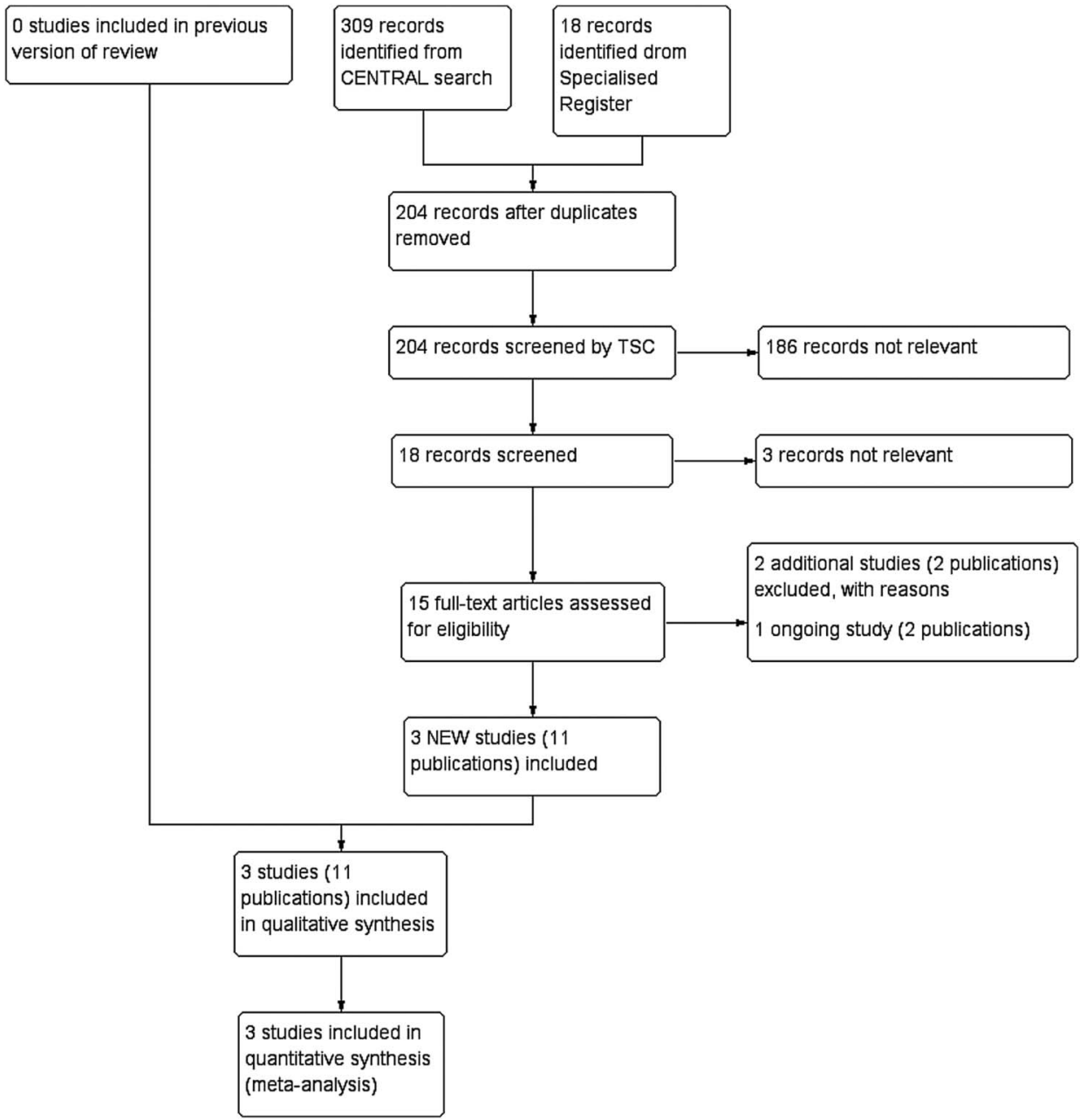


achieving a seal and haemodynamic stability. However, the use of bifurcated stent graft is routine for ruptured AAA. Early reports have suggested a trend towards reduction in perioperative morbidity and mortality in selected patients. Indeed evidence is emerging that the inflammatory response to RAAA repair and the associated organ dysfunction is attenuated by eEVAR compared with open repair. ${ }^{31} 32$

The purpose of this review was to assess the available evidence to support the use of eEVAR to treat RAAA. The advantages and disadvantages of eEVAR for patients with RAAA was determined by the effect on short-term mortality, major complication rates, aneurysm exclusion and late complications when compared with patients who have had conventional open repair of RAAA. The results of this review have been published as a Cochrane review, which was an update of the initial study. ${ }^{33} 34$

\section{METHODS}

Only prospective RCTs comparing eEVAR with conventional open surgical repair were considered eligible for inclusion. Within the studies, all patients in whom a RAAA has been clinically diagnosed by CT, angiography, MR angiography or objective acute symptoms suggestive of impending rupture of the aneurysm to warrant inclusion. All types of endovascular devices were considered in comparison with conventional open surgical treatment for patients considered fit for surgery.

The primary outcome for the study was the short-term morality (30-day, or in-hospital mortality). Secondary outcomes included:

- Aneurysm exclusion, or further extravasation beyond the sac on follow-up imaging 30 days after the procedure;

- Major complications, such as open conversion, haemorrhage, myocardial infarction, stroke, renal failure $(20 \%$ rise in creatinine levels), respiratory failure (requirement for postoperative mechanical ventilation), pneumonia, bowel ischaemia, lower limb ischaemia;

- Minor complications, such as catheter site haematoma, wound infection;

- Long-term complications and mortality; reintervention rates for problems related to the RAAA or its treatment will be sought where possible, as will cause of death, with or without reintervention, that is, device related;

- Quality of life (standardised questionnaires);

- Economic analysis (cost per patient).

\section{SEARCH METHODS}

For this update, the Cochrane Peripheral Vascular Diseases Group Trials Search Co-ordinator (TSC) searched the Specialised Register (last searched February 2014) and the Cochrane Central Register of Controlled Trials (CENTRAL) 2014, Issue 2, part of The Cochrane Library. ${ }^{35}$ The Specialised Register is maintained by the TSC and is constructed from weekly electronic searches of MEDLINE, EMBASE, CINAHL, AMED and through hand searching relevant journals. The full list of the databases, journals and conference proceedings which have been searched, as well as the search strategies used are described in the Specialised Register section of the Cochrane Peripheral Vascular Diseases Group module in The Cochrane Library. ${ }^{35} 36$ References of relevant studies were reviewed for other pertinent publications.

\section{Data collection and extraction}

Two authors ( $\mathrm{SAB}$ and $\mathrm{RB}$ ) independently reviewed the studies identified by the search for their relevance using the selection criteria. Disagreements were resolved through discussion. Two review authors ( $\mathrm{SAB}$ and $\mathrm{RB}$ ) independently extracted data for each included study.

Table 1 The string search performed of the terms in the literature

\begin{tabular}{llr}
\hline$\# 1$ & MeSH descriptor: [Ednovascular & 6017 \\
& Procedures] explode all trees & \\
$\# 2$ & MeSH descriptor: [Stents] explode all trees & 3314 \\
$\# 3$ & MeSH descriptor: [Vascular Surgical & 652 \\
& Procedures] this term only & \\
$\# 4$ & Endovasc ${ }^{\star}:$ ti,ab,kw & 941 \\
$\# 5$ & Endostent*:ti,ab,kw & 1 \\
$\# 6$ & Endograft* & 81 \\
$\# 7$ & EVRAR:ti,ab,kw & 1 \\
$\# 8$ & (EVAR or REVAR):ti,ab,kw & 74 \\
$\# 9$ & (Palmaz):ti,ab,kw & 91 \\
$\# 10$ & ${\text { "stent }{ }^{*} \text { or graft* }}^{*}$ & 5489 \\
$\# 11$ & Palmaz:ti,ab,kw & 91 \\
$\# 12$ & Viabahn or Nitinol or Hemobahn or Intracoil & 207 \\
& or Tantalum or powerlink or excluder or & \\
& talent or aorfix or endologix or anaconda:ti, & \\
& ab,kw &
\end{tabular}

\#13 Zenith or Dynalink or Hemobahn or Luminex or Memotherm or Wallstent:ti,ab, kw

\#14 MeSH descriptor: [Blood Vessel Prosthesis] explode all terms

\#15 MeSH descriptor: \{Blood Vessel Prosthesis Implantation] this term only

\#16 Endoprosthesis:ti,ab,kw

\#17 \#1 or \#2 or \#3 or \#4 or \#5 or \#6 or \#7 or \#8 or \#9 or \#10 or \#11 or \#12 or \#13 or \#14 or \#15 or \#16

\#18 MeSH descriptor: [Aneurysm, Ruptured] explode all terms

\#19 MeSH descriptor: [Aneurysm, Dissecting] this term only

\#20 (aneurysm* or abdom* or thoracoabdom* or thorac-abdom* or aort*) near (ruptur* or tear or bleed* or trauma)

\#21 MeSH descriptor: [Aorta] explode all trees and with qualifier(s): [Surgery-SU]

\#22 RAAA

\#23 \#18 or \#19 or \#20 or \#21 or \#22

\#24 \#17 and \#23 in Trials 
Table 2 The details of the Hinchliffe study

Methods

Participants

Interventions

\section{Outcomes}

Notes

Risk of bias

Bias

Random sequence generation (selection bias

\section{Allocation concealment}

(selection bias)

Blinding of participants and

personnel (performance bias);

all outcomes

Blinding of outcome

assessment (detection bias); all

outcomes

Incomplete outcome data

(attrition bias); all outcomes

Selective reporting (reporting

bias)

Other bias
Study type: single-centre, randomised controlled trial, open label, intention-to-treat Study aim: to test the hypothesis that EVAR can reduce the perioperative mortality of ruptured AAA, compared with open repair

Country: England

Setting: hospital

Number randomised: total $n=32$ (eEVAR $n=15$; open repair $n=17$ )

Age (median years (IQR)): eEVAR=74 years (68.8-79.5); open repair=80 years (73. 8-83.8)

Gender (M/F): eEVAR=11/4; open repair=13/4

Inclusion criteria: all patients admitted with clinically suspected or radiologically confirmed rupture of an infrarenal abdominal aortic aneurysm that, in the opinion of the duty consultant vascular surgeon would normally be treated with open repair

Exclusion criteria: no endovascular team available; full selection of emergency stent grafts not available; age $<50$ years; inability to give verbal or written consent; unconscious patient; allergy to radiological contrast, stainless steel or polyester; severe comorbidity that would preclude intensive care treatment following open repair; previous endovascular AAA repair; women of childbearing potential not taking contraception; pregnant and lactating women eEVAR anatomical suitability (exclusion criteria): absolute contraindications: no evidence on aneurysm rupture, juxtarenal aneurysm, neck diameter $>32 \mathrm{~mm}$, external iliac artery diameter $>6 \mathrm{~mm}$; relative contraindications: proximal neck length $<10 \mathrm{~mm}$, excessive thrombus in the proximal neck, common iliac artery length $<25 \mathrm{~mm}$, heavily calcified iliac arteries

eEVAR description: those with a diagnostic CT were transferred directly to operating theatre, and those without first had a CT scan to determine aortic measurement; performed in dedicated vascular operating theatre using a Siremobil 2000 image intensifier, with digital subtraction angiography facilities; most patients heparinised; two-piece aortouniiliac stent graft made with Gianturco stents with uncovered suprarenal component; occluding device used in contralateral common iliac artery; after deployment of stent graft, a femoro-femoral crossover graft was performed

Open repair description: after randomisation to open repair, patients were transferred directly to the operating theatre, according to local practice; performed transperitoneally either by midline or transverse incisions; aorta clamped below renal arteries; patients no heparinised; inlay technique was used and grafts were gelatin-coated polyester Perioperative mortality, defined as 30-day or in-hospital

'Patients were deemed suitable for EVAR if, in the opinion of the operating surgeon, they could perform the repair'; participants recruited September 2002 to December 2004; five surgeons on unit, required that surgeon and team available had sufficient expertise to offer EVAR, if not, conventional open repair was offered; unstable patients that might be disadvantaged by delay incurred by CT scan could, at the surgeon's discretion, not be randomised and taken directly for open repair

Authors' Support for judgement judgement

Unclear 'Randomisation was then performed from sealed opaque envelopes kept in the risk Accident and Emergency Department'. Unclear how randomisation sequence was generated

Low risk 'Randomisation was then performed from sealed opaque envelopes kept in the Accident and Emergency Department'

Low risk Study was unblinded, due to nature of intervention but unlikely to influence outcomes. 'The surgeons were blinded to the dimensions of patient's aorta until randomisation had taken place' to avoid bias

Low risk Not possible to blind team regarding allocation group, but unlikely to influence outcome measures

Low risk All patients accounted for; crossover patients accounted for; similar dropout rates and reasons between treatment groups

Low risk Most of the protocol outlined in the text; all relevant outcomes reported; with the exception of mortality, outcomes are not well described in the methods

Unclear Underpowered study: 32 of the required 100 patients recruited

risk

AAA, abdominal aortic aneurysm; eEVAR, emergency endovascular aneurysm repair; $F$, female; M, male. 
Table 3 The details of the Amsterdam Acute Aneurysm Trial (AJAX) study

\begin{tabular}{|c|c|}
\hline Methods & $\begin{array}{l}\text { Study type: multicentre, randomised controlled trial, intention-to-treat } \\
\text { Study aim: compare EVAR and open repair in treating RAAA on mortality and } \\
\text { severe complications } \\
\text { Country: The Netherlands } \\
\text { Setting: three large hospital vascular centres in Amsterdam }\end{array}$ \\
\hline Participants & 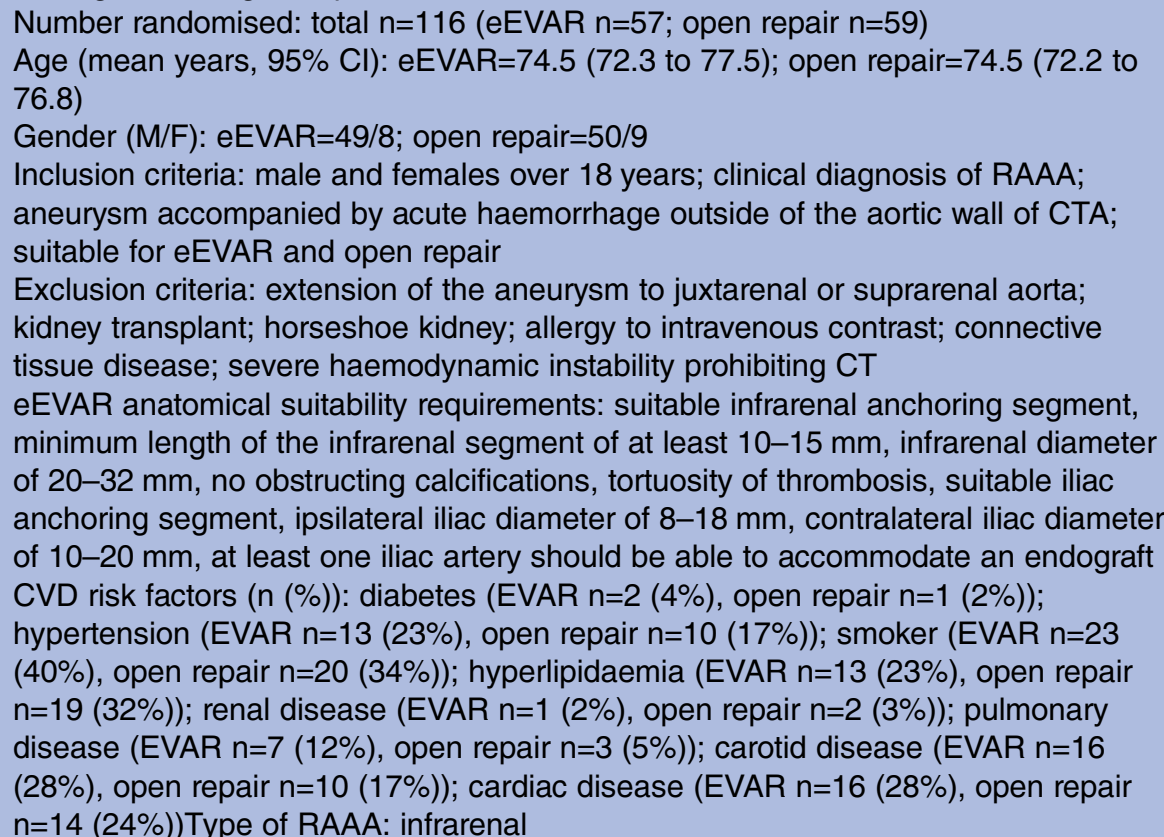 \\
\hline Interventions & $\begin{array}{l}\text { eEVAR description: aortouniiliac endograft and contralateral iliac occluding device, } \\
\text { followed by a femoro-femoral crossover bypass graft } \\
\text { Open repair description: midline laparotomy and exclusion of rupture aneurysm by } \\
\text { either polyester tube or bifurcated graft; conducted under general anaesthesia }\end{array}$ \\
\hline Outcomes & $\begin{array}{l}\text { Composite death and severe complications at } 30 \text { days after intervention; long-term } \\
\text { mortality rates ( } 6 \text { months after randomisation); length of hospital and ICU stay; } \\
\text { duration of intubation/ventilation; use of blood products; for EVAR, occurrence of } \\
\text { endoleaks }\end{array}$ \\
\hline Notes & $\begin{array}{l}\text { Study period: April } 2004 \text { to February } 2011 \text {; three main trial centres, all other (seven) } \\
\text { regional hospitals transferred participants to one of the trial centres }\end{array}$ \\
\hline
\end{tabular}

Risk of bias

Bias

Authors' judgement

Random sequence generation Low risk

\section{Support for judgement}

(selection bias)

clinical research unit using ALEA software for randomization in clinical trials with a $1: 1$ allocation using random block sizes of 4 or 6 , stratified for each participating centre'.

\section{Allocation concealment (selection bias) Low risk}

Blinding of participants and personnel (performance bias); all outcomes Blinding of outcome assessment (detection bias); all outcomes Incomplete outcome data (attrition bias); all outcomes

Selective reporting (reporting bias)
Low risk

Low risk

Low risk

Unclear risk 'Allocation was concealed using sequentially numbered opaque sealed envelopes'.

Surgical team not possible to blind, but unlikely to influence outcomes

Double database entry; end point adjudication committee blinded; independent safety committee blinded

All patients accounted for in CONSORT diagram; both treatment groups had similar dropout rates and reasons

Although all predescribed outcomes listed in the Reimerink publication are reported, quality of life and cost-effectiveness as described in the study protocol, were not included, suggesting further publications to emerge None 
Table 4 The details of the Immediate Management of the Patient with Rupture: Open Versus Endovascular (IMPROVE) study

Methods

Participants

Interventions

\section{Outcomes}

Notes

Risk of bias

Bias

Random sequence generation (selection bias)

Allocation concealment

(selection bias)

Blinding of participants and

personnel (performance bias);

all outcomes

Blinding of outcome

assessment (detection bias);

all outcomes

Incomplete outcome data

(attrition bias); all outcomes

Selective reporting (reporting

bias)

Other bias

CVD, cardiovascular disease; eEVAR, emergency endovascular aneurysm repair; F, female; M, male; RAAA, ruptured abdominal aortic aneurysm.

Details about the trial design, characteristics of participants, diagnosis of RAAA, eEVAR and open repair procedures were recorded. Data were collected on the primary outcome short-term mortality (30-day or in-hospital) and secondary outcomes: aneurysm
Study type: multicentre, randomised controlled trial, open label, intention-to-treat Study aim: to assess whether EVAR vs open repair reduces early mortality for patients with suspected RAAA

Country: UK and Canada

Setting: 30 hospital vascular units and specialist centres

Number randomised: total $n=613$ (eEVAR $n=316$; open repair $n=297$ )

Age (mean years $( \pm$ SD)): eEVAR=76.7 (7.4); open repair=76.7 (7.8)

Gender (M/F): eEVAR=246/70; open repair=234/63

Inclusion criteria: men and women over the age of 50 years; clinical diagnosis of RAAA or ruptured aortoiliac aneurysm, made by a senior trial hospital clinician

Exclusion criteria: previous aneurysm repair; rupture of an isolated internal iliac aneurysm, aortocaval or aortoenteric fistulae; recent anatomical assessment of the aorta; connective tissue disorder; if intervention was considered futile

eEVAR anatomical suitability requirements: no absolute requirements will be set for the study, but proximal neck morphology with a diameter exceeding $32 \mathrm{~mm}$ or a length less than $10 \mathrm{~mm}$ may be considered unfavourable, and iliac artery diameters should be in the range of 8-22 mm

CVD risk factors ( $n(\%))$ : not given

Type of RAAA: 'ruptured AAA or ruptured aortoiliac aneurysm'

eEVAR description: endovascular supracoeliac aortic balloon occlusion will be used to support less stable patients; most interventions performed with aortouniiliac graft, but some patients received bifurcated grafts, with subsequent femoro-femoral crossover graft with contralateral iliac occlusion; control of aorta achieved using local/region anaesthesia, with general anaesthesia used later in procedure if necessary Open repair description: CT scan is optional; aneurysms repaired by cross-clamping the proximal aorta and inserting a prosthetic inlay graft; performed under general anaesthesia

30-day mortality, $24 \mathrm{~h}$ and in-hospital mortality, costs, reinterventions at primary admission time and place of discharge; cost-effectiveness and mortality at 12 months are planned for future reporting

Participants recruited September 2009 to July 2013; flow diagram shows 623 randomised, but 10 were excluded after Data Monitoring Committee reviewed participants, 613 used in analysis; only $275(87 \%)$ of EVAR and 261 (88\%) of open repair had confirmed RAAA

\section{Authors' Support for judgement}

judgement

Low risk

'And independent contractor provided telephone randomisation, with computer-generated assignation of patients in a 1:1 ratio, using variable block size and stratified by centre'.

Low risk 'And independent contractor provided telephone randomisation, with computer generated assignation of patients...'

Low risk Surgical team not possible to blind, but unlikely to influence outcomes

Low risk Data verification performed centrally at the trial core laboratory, unclear if blinding, but unlikely to influence outcomes

Low risk All patients accounted for, with both treatment groups having similar dropout rates/reasoning

Unclear risk All predescribed outcomes reported on, but not all appropriate outcomes reported yet, suggesting further publications to emerge

\section{Low risk None}


Figure 2 Short-term mortality (30-day or in-hospital) of emergency endovascular aneurysm repair (eEVAR) versus open repair.

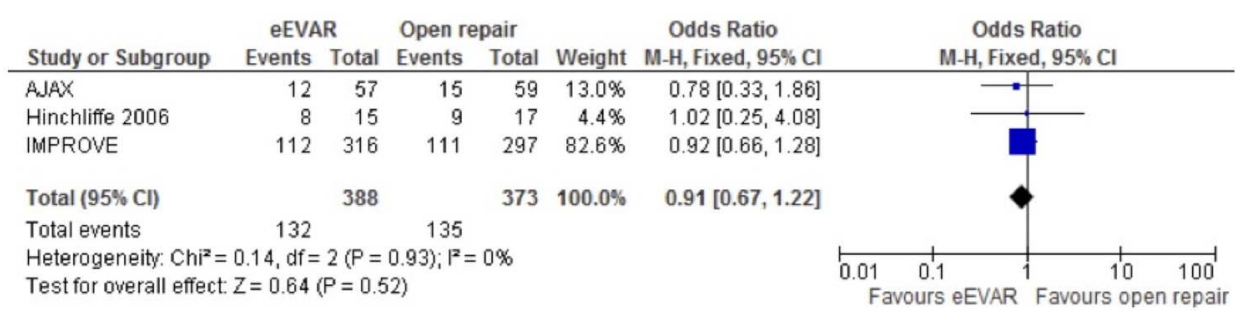

Acute Aneurysm Trial (AJAX) and Hinchliffe trials only used aortouniiliac devices, while the Immediate Management of the Patient with Rupture: Open Versus Endovascular (IMPROVE) used both methods, with a greater proportion being aortobiiliac. ${ }^{38-48}$ However, due to a paucity of information, subgroup analysis was not possible at this time.

\section{Sensitivity analysis}

Although all the participants in the IMPROVE trial had a clinical diagnoses of RAAA, on start of the intervention, it was found that only $536(87 \%)$ of the 613 randomised participants had, in fact, a ruptured AAA. ${ }^{46}{ }^{48}$ Of the remaining 77 participants, 22 had symptomatic nonruptured AAA, or other final diagnoses. Sensitivity analyses were planned to evaluate the effects of this trial on the outcomes. into meta-analysis and ORs with $95 \%$ CIs were to be calculated. For continuous data, meta-analysis would provide mean differences with SDs.

\section{Assessment of heterogeneity}

A test for heterogeneity examines the null hypothesis that all studies are evaluating the same effect. We obtained $\mathrm{p}$ values comparing the test statistic with a $\chi^{2}$ distribution. A measure $\left(\mathrm{I}^{2}\right)$ that describes the percentage of total variation across studies due to heterogeneity rather than by chance was included. A value of $0 \%$ indicates no observed heterogeneity, and larger values show increasing heterogeneity. ${ }^{37}$

\section{Data synthesis}

Data extracted independently by two review authors (SAB and $\mathrm{RB}$ ) was compiled and entered into RevMan by one author (RB). Comparisons of data using meta-analyses were undertaken using fixed-effects models, unless the test for heterogeneity yielded a value $>50 \%$, in which case a random-effects model was implemented.

\section{Subgroup analysis and investigation of heterogeneity}

We planned to perform subgroup analysis to evaluate the impact of patients treated with aortouniiliac devices and those treated with aortobiiliac devices. The Amsterdam
Figure 3 Myocardial infarction of emergency endovascular aneurysm repair (eEVAR) versus open repair.

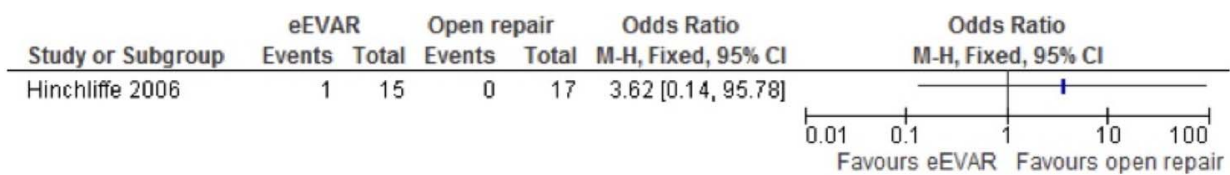




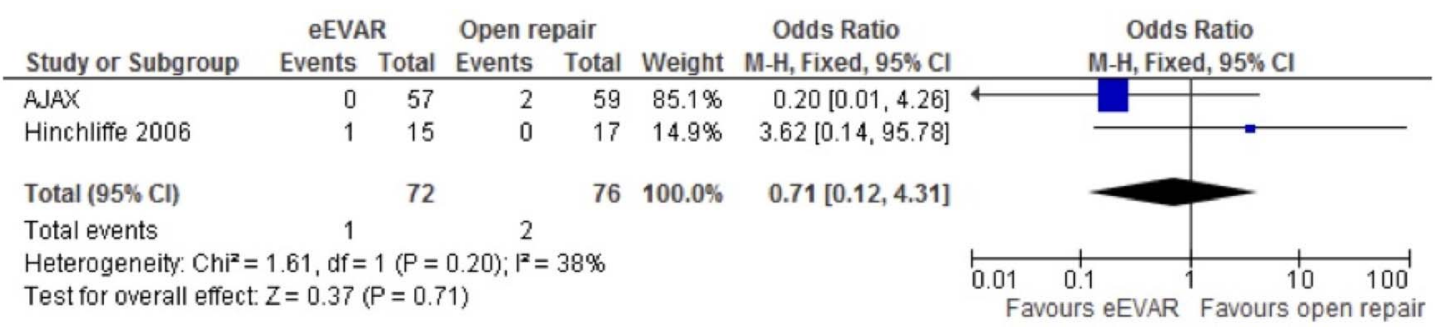

Figure 4 Stroke complication of emergency endovascular aneurysm repair (eEVAR) versus open repair.

AJAX and IMPROVE were both multicentre studies, with AJAX taking place in Amsterdam, the Netherlands, and IMPROVE in the UK, with one study site in Canada. Hinchliffe $e t$ al was a single-centre trial within England. All included participants had a clinical diagnosis of RAAA, but in the IMPROVE study only 536 out of the 613 $(87 \%)$ randomised participants actually had RAAA, the remaining 77 had symptomatic non-ruptured AAA (22), no AAA (10), or asymptomatic AAA or another final diagnosis (45). For the AJAX study, all randomised participants were considered suitable for both eEVAR and open repair, which in the Hinchliffe and IMPROVE studies suitability for eEVAR was determined after randomisation. In the Hinchliffe and AJAX studies, aortouniiliac grafts were used in the endovascular trial arm, while the IMPROVE trial used both this and, more predominately, aortobiiliac grafts. The data derived from the three studies are outlined in tables 2-4 with quality of data described.

Five studies were excluded from this review. Three studies were prospective trials, treating patients presenting with RAAA with eEVAR. ${ }^{29} 5455$ However, their comparison to open repair was made through retrospective, 'historical controls' or with open repair cohorts. One study was a prospective comparison between eEVAR and open repair in patients with RAAA, but the study was non-randomised. ${ }^{49} \mathrm{~A}$ final study was a non-randomised study of 55 consecutive patients presenting with RAAA. ${ }^{50}$ A portion of the participants in the study were collected retrospectively and a portion prospectively.

\section{Allocation (selection bias)}

Both the AJAX and IMPROVE studies adequately reported random sequence generation, but the Hinchliffe study did not give a description of how their sequence was produced, and is therefore at unclear risk. For allocation concealment, all three studies clearly explained adequate concealment methods.

\section{Blinding (performance bias and detection bias)}

Owing to the nature of the intervention, it is not possible to blind the surgeons and difficult to blind patients and the research team to the treatment allocation. However, we determined that a lack of blinding is unlikely to influence the outcomes of interest, and all three have been listed as low risk for performance and detection bias. Attempts were made to reduce the risk of bias by the study authors: in the AJAX study, an end point adjudication committee and independent safety committee, both blinded to treatment allocation, were utilised. The Hinchliffe study kept surgeons blinded to dimensions of the aorta until randomisation was completed, and the IMPROVE study utilised a trial core laboratory to centrally verify outcomes.

\section{Incomplete outcome data (attrition bias) and selective reporting (reporting bias)}

All three included studies adequately accounted for all participants, giving thorough explanation of all dropout rates and reasons. All three studies were at a low risk of attrition bias. Both the AJAX and IMPROVE trials are lacking important outcome data specified in their protocols, which include quality of life and costs for both studies, and complications and long-term survival for the IMPROVE study. It is assumed that these outcomes will be reported in future publications, but until that data are reported, the two studies are at an unclear risk of reporting bias. The Hinchliffe study reported on all outcomes specified.

\section{Other potential sources of bias}

The AJAX and IMPROVE studies appear to be free of other sources of bias, but the Hinchliffe study could be at risk of bias due to an underpowered study population. In the publication, it was reported that the study

\begin{tabular}{|c|c|c|c|c|c|c|c|c|}
\hline \multirow[b]{2}{*}{ Study or Subgroup } & \multicolumn{2}{|c|}{ eEVAR } & \multicolumn{2}{|c|}{ Open repair } & \multirow[b]{2}{*}{ Weight } & \multirow{2}{*}{$\begin{array}{l}\text { Odds Ratio } \\
\text { M-H, Fixed, } 95 \% \mathrm{Cl}\end{array}$} & \multirow{2}{*}{$\begin{array}{l}\text { Odds Ratio } \\
\text { M-H, Fixed, } 95 \% \mathrm{Cl}\end{array}$} & \\
\hline & Events & Total & Events & Total & & & & \\
\hline AJAAX & 4 & 57 & 2 & 57 & $29.8 \%$ & $2.08[0.36,11.81]$ & 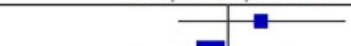 & \\
\hline Hinchliffe 2006 & 5 & 15 & 7 & 17 & $70.2 \%$ & $0.71[0.17,3.03]$ & & \\
\hline Total $(95 \% \mathrm{Cl})$ & & 72 & & 74 & $100.0 \%$ & $1.12[0.38,3.30]$ & & \\
\hline Total events & 9 & & 9 & & & & & \\
\hline \multicolumn{7}{|c|}{$\begin{array}{l}\text { Heterogeneity: } \mathrm{Chi}^{2}=0.86, \mathrm{df}=1(P=0.35) ;\left.\right|^{2}=0 \% \\
\text { Test for overall effect: } Z=0.21(P=0.84)\end{array}$} & $\begin{array}{lll}0.01 & 0.1 & 1\end{array}$ & $\begin{array}{c}100 \\
\text { repair }\end{array}$ \\
\hline
\end{tabular}

Figure 5 Cardiac complication (moderate or severe) of emergency endovascular aneurysm repair (eEVAR) versus open repair. 


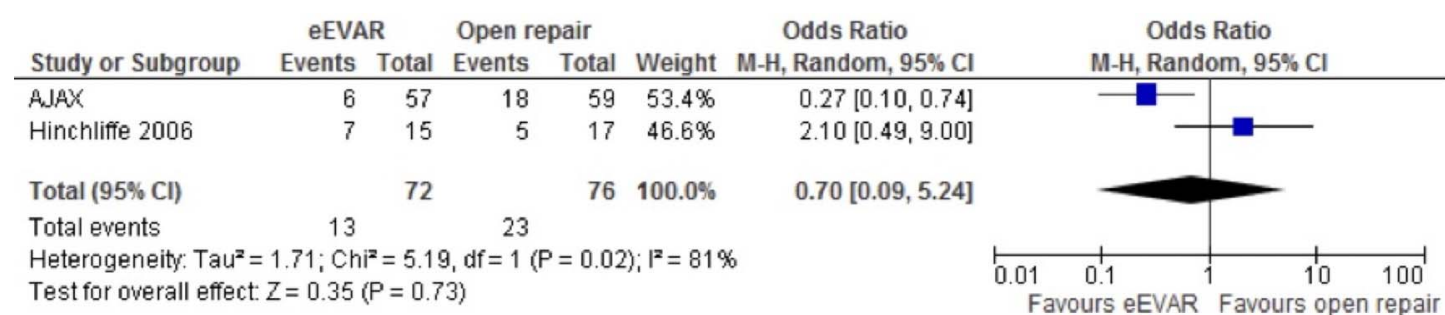

Figure 6 Renal complications (moderate or severe) of emergency endovascular aneurysm repair (eEVAR) versus open repair.

required 100 participants to be adequately powered, yet they only included 32 patients.

\section{EFFECTS OF INTERVENTIONS}

Mortality (30-day or in-hospital)

All three studies were included in the meta-analysis for mortality (30-day or in-hospital). The fixed-effects model found no clear evidence to support a difference in mortality between eEVAR and open repair, OR 0.93 (95\% CI 0.69 to $1.25 ; \mathrm{p}=0.63$; figure 2 ). When the IMPROVE study was removed for sensitivity analysis, due to randomisation of patients that did not have RAAA, the OR moved closer to one and the CI became wider as the IMPROVE study had a larger study population than the other two included studies, OR 0.98 (95\% CI 0.49 to $1.95 ; \mathrm{p}=0.95$ ).

\section{Complications (30-day)}

Myocardial infarction

Only the Hinchliffe study reported myocardial infarction, but only one event was reported so the CI is very wide, OR 3.62 (95\% CI 0.14 to 95.78; figure 3). As only one study was included for this outcome, no overall association could be determined, and the wide CI makes any conclusion spurious.

\section{Stroke}

Both the AJAX and Hinchliffe studies reported stroke events, but with very few events, and opposing findings, the fixed-effects model, with a non-significant OR has a very wide CI that is difficult to derive any meaningful conclusion from (OR $0.71,95 \%$ CI 0.12 to 4.31 ; $\mathrm{p}=0.71$; figure 4).

\section{Cardiac complications (moderate or severe)}

Cardiac complications were evaluated in the AJAX and Hinchliffe studies. The fixed-effects meta-analysis found a non-significant OR, but again with a wide CI (OR 1.12, $95 \%$ CI 0.38 to $3.30 ; \mathrm{p}=0.84$; figure 5 ).

\section{Renal complications (moderate or severe)}

The AJAX and Hinchliffe studies reported renal complications, and the random-effects model used to analyse the association found no clear difference between the interventions, OR 0.70 (95\% CI 0.09 to $5.24 ; \mathrm{p}=0.73$; figure 6).

\section{Severe bowel ischaemia}

Only a single study evaluated severe bowel ischaemia (AJAX), which had an OR of 0.39 (95\% CI 0.07 to 2.11 ), but as only one study was included, no overall association could be determined (figure 7 ).

\section{Spinal cord ischaemia}

Spinal cord ischaemia was also only evaluated in the AJAX study, which had only one event. With an OR of 3.16 and a $95 \%$ CI of 0.13 to 79.17 , very little can currently be concluded regarding this outcome (figure 8).

\section{Reoperation}

The occurrence of reoperation, specific to the aneurysm repair, was reported in two studies (AJAX and Hinchliffe). The fixed-effects model did not find clear evidence to support a difference between the interventions, OR 0.89 (95\% CI 0.39 to $2.01 ; \mathrm{p}=0.78)$, again with a rather wide CI (figure 9).

\section{Amputation}

The AJAX trial was the only study to evaluate amputation, and with the few events, all in the open repair intervention group, the OR of 0.14 (95\% CI 0.01 to 2.78) had a wide CI and no association to be concluded, as only a single study was included (figure 10).

\section{Respiratory failure}

Respiratory failure was evaluated in the Hinchliffe study, alone. With only a single event in the eEVAR arm, the CI was very wide, OR 3.62 (95\% CI 0.14 to 95.78 ) with no overall association (figure 11).

\begin{tabular}{|c|c|c|c|c|c|c|c|c|c|}
\hline \multirow[b]{2}{*}{ Study or Subgroup } & \multicolumn{2}{|c|}{ eEVAR } & \multicolumn{2}{|c|}{ Open repair } & \multirow{2}{*}{$\begin{array}{c}\text { Odds Ratio } \\
\text { M-H, Fixed, 95\% Cl }\end{array}$} & \multirow{2}{*}{\multicolumn{3}{|c|}{$\begin{array}{c}\text { Odds Ratio } \\
\text { M-H, Fixed, 95\% Cl }\end{array}$}} & \\
\hline & Events & Total & Events & Total & & & & & \\
\hline AJAXX & 2 & 57 & 5 & 59 & $0.39[0.07,2.11]$ & & 1 & E & \\
\hline & & & & & & $\begin{array}{l}0.01 \\
\mathrm{FaI}\end{array}$ & $\begin{array}{l}0.1 \\
\text { urs eEVAR }\end{array}$ & $\begin{array}{r}10 \\
\text { Favours of }\end{array}$ & $\begin{array}{c}100 \\
\text { n repair }\end{array}$ \\
\hline
\end{tabular}

Figure 7 Bowel ischaemia of emergency endovascular aneurysm repair (eEVAR) versus open repair. 
Figure 8 Spinal cord ischaemia of emergency endovascular aneurysm repair (eEVAR) versus open repair.

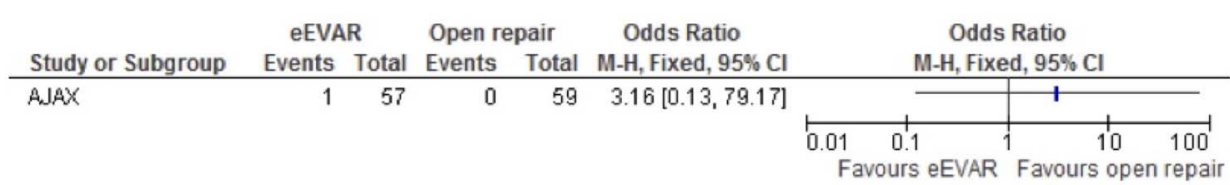

\section{Long-term outcomes (6 months)}

Long-term outcomes were only evaluated in the AJAX study. There was no clear evidence to support a difference between the interventions for mortality at 6 months in the eEVAR arm (OR $0.89,95 \%$ CI 0.40 to 1.98; figure 12), or long-term reoperation (OR 1.28, $95 \%$ CI 0.53 to 3.06; figure 13 ). No conclusions could be drawn from the single study.

\section{Cost per patient (30-day)}

Cost per patient was only evaluated in the IMPROVE study, which found the mean cost slightly less in the eEVAR treated arm after 30 days, £13 433 compared with $£ 14619$ in the open repair group. The mean difference worked out to be $£ 1186$, favouring eEVAR, but as both trial arms had large SDs, the 95\% CI was very wide, spanning £-2996.24 to £624.24. As only a single study reported on cost, no overall association could be determined (figure 14).

\section{Open conversion}

As open conversion can only be evaluated in the eEVAR treatment group, meta-analysis is not an appropriate way to compare this outcome between the three studies. The AJAX reported 10 cases of open conversion in the 57 $(17.5 \%)$ participants randomised to eEVAR. Hinchliffe had 1 open conversions out of the $15(6.7 \%)$ randomised to eEVAR, and the IMPROVE study only had 4 out of $316(1.3 \%)$ randomised, which is far lower than the other two trials. This could also be the result of the $13 \%$ of randomised participants in the IMPROVE study that did not have RAAA, but rather 22 participants had symptomatic non-ruptured AAA, 10 had no AAA and 45 had asymptomatic AAA or other final diagnoses and also 84 participants randomised to eEVAR were determined unsuitable for the procedure and moved to open repair, but were not considered open conversion.

Table 5 contains perioperative and postoperative patient characteristics that were not considered as outcomes in this review, but are of interest when comparing
eEVAR with open repair, and also for comparisons between the trials. The table addresses time spent waiting for surgical intervention, time in operating theatre, blood loss during operation and length of time spent in hospital. As two studies used median and IQR and one study reported using mean and SD, the findings could not be compared quantitatively, but rather used for anecdotal analysis.

\section{DISCUSSION}

The three studies eligible for inclusion in this review were of good quality. The evidence gathered was relevant but there were few data to support an association, and other outcomes of interest were not acknowledged within the studies, such as minor complications and quality of life. Although all potential sources of data were carefully vetted, it remains possible that relevant data were not published, or that were not found in the search.

All three included studies did require a clinical diagnosis of RAAA for inclusion in the study, yet the IMPROVE study, on start of intervention, found that 77 (13\%) of their included, randomised participants did not have RAAA; 22 had symptomatic non-ruptured AAA, 10 had no AAA and 45 had asymptomatic AAA or another final diagnosis. The authors of the IMPROVE trial claimed this method was a more 'real-world' approach to the issue. While this may not effect the overall outcomes, it is of concern. Also, the IMPROVE trial did not assess eEVAR suitability prior to randomisation, which resulted in 84 participants randomised to eEVAR not being suitable for the procedure and transferred to open repair. Hinchliffe $e t$ al also did not select participants for their suitability for both eEVAR and open repair prior to randomisation, and one patient randomised to eEVAR was transferred to open repair. The AJAX trial evaluated a more selected study population of participants suitable for both eEVAR and open repair. These methodological differences could possibly alter the applicability of the evidence as the results could be aimed at different populations.

\begin{tabular}{|c|c|c|c|c|c|c|c|c|}
\hline \multirow[b]{2}{*}{ Study or Subgroup } & \multicolumn{2}{|c|}{ eEVAR } & \multicolumn{2}{|c|}{ Open repair } & \multicolumn{2}{|r|}{ Odds Ratio } & \multirow{2}{*}{$\begin{array}{c}\text { Odds Ratio } \\
\text { M-H, Fixed, } 95 \% \mathrm{Cl}\end{array}$} & \\
\hline & Events & Total & Events & Total & Weight & M-H, Fixed, $95 \%$ Cl & & \\
\hline AJIAX & 13 & 57 & 12 & 59 & $74.0 \%$ & $1.16[0.48,2.81]$ & & \\
\hline Hinchliffe 2006 & 0 & 15 & 3 & 17 & $26.0 \%$ & $0.13[0.01,2.82]$ & 4 & \\
\hline Total $(95 \% \mathrm{Cl})$ & & 72 & & 76 & $100.0 \%$ & $0.89[0.39,2.01]$ & & \\
\hline Total events & 13 & & 15 & & & & & \\
\hline $\begin{array}{l}\text { Heterogeneity: } \mathrm{Chi}^{2}= \\
\text { Test for overall effect }\end{array}$ & $\begin{array}{l}1.82, d f= \\
Z=0.28\end{array}$ & $\begin{array}{l}1(P= \\
(P=0.7\end{array}$ & $\begin{array}{l}0.18) ;\left.\right|^{2}= \\
88)\end{array}$ & $45 \%$ & & & $\begin{array}{llll}0.01 & 0.1 & 1 & 1 \\
& \text { Favours eEVAR } & \text { Favours }\end{array}$ & $\begin{array}{ll}10 & 100 \\
5 & \text { open repair }\end{array}$ \\
\hline
\end{tabular}

Figure 9 Reoperation of emergency endovascular aneurysm repair (eEVAR) versus open repair. 


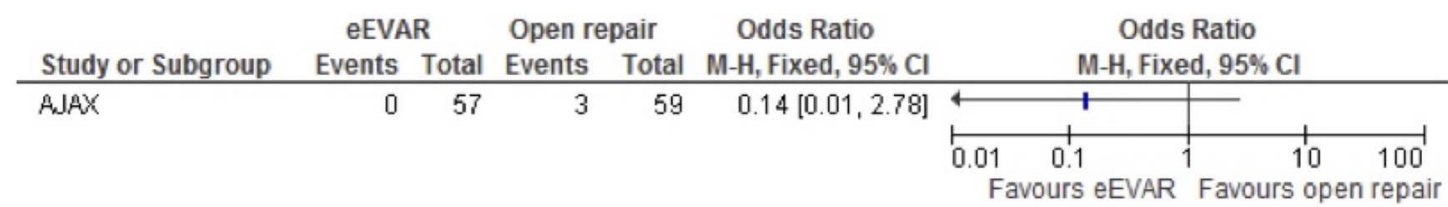

Figure 10 Amputation after emergency endovascular aneurysm repair (eEVAR) versus open repair.

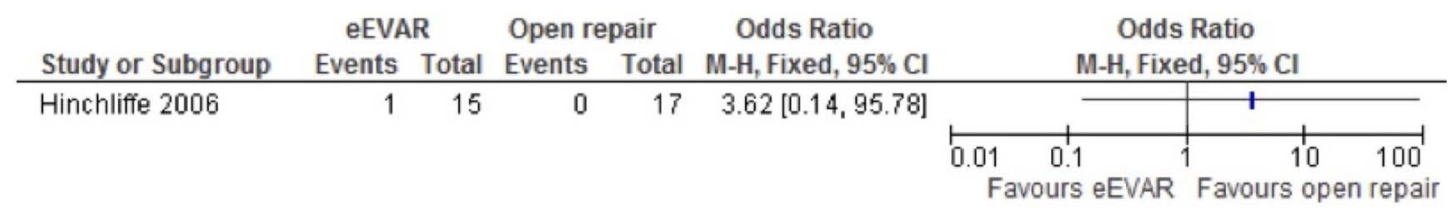

Figure 11 Respiratory failure of emergency endovascular aneurysm repair (eEVAR) versus open repair.

\begin{tabular}{|c|c|c|c|c|c|c|c|c|}
\hline \multirow[b]{2}{*}{ Study or Subgroup } & \multicolumn{2}{|c|}{ eEVAR } & \multicolumn{2}{|c|}{ Open repair } & \multirow{2}{*}{$\begin{array}{c}\text { Odds Ratio } \\
\text { M-H, Fixed, } 95 \% \mathrm{Cl}\end{array}$} & \multirow{2}{*}{\multicolumn{3}{|c|}{$\begin{array}{c}\text { Odds Ratio } \\
\text { M-H, Fixed, } 95 \% \mathrm{Cl}\end{array}$}} \\
\hline & Events & Total & Events & Total & & & & \\
\hline AJIAX & 16 & 57 & 18 & 59 & $0.89[0.40,1.98]$ & $\longrightarrow$ & 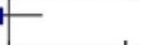 & \\
\hline & & & & & & $\begin{array}{l}0.010 .1 \\
\text { Favours eEVAR }\end{array}$ & $\begin{array}{r}10 \\
\text { Favours o }\end{array}$ & $\begin{array}{r}100 \\
\text { n repair }\end{array}$ \\
\hline
\end{tabular}

Figure 12 Mortality at 6 month for emergency endovascular aneurysm repair (eEVAR) versus open repair.

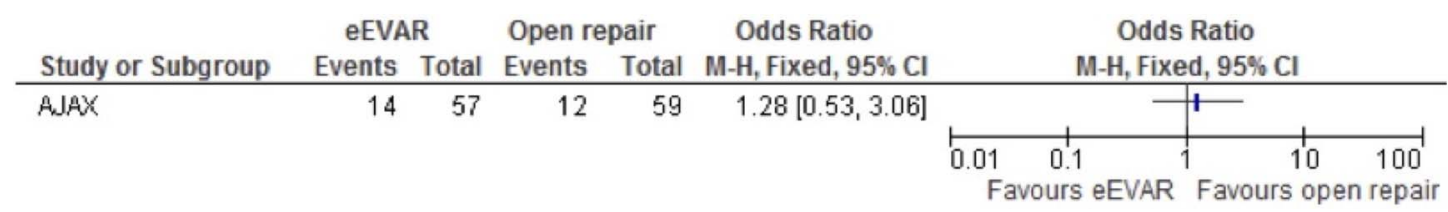

Figure 13 Reoperation at 6 months for emergency endovascular aneurysm repair (eEVAR) versus open repair.

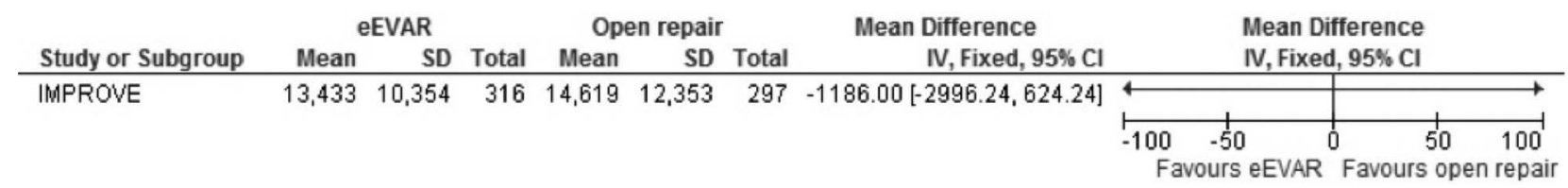

Figure 14 Cost per patient (30 days) of emergency endovascular aneurysm repair (eEVAR) versus open repair.

Table 5 The perioperative details of the three studies

\begin{tabular}{|c|c|c|c|}
\hline & AJAX (median, IQR) & Hinchliffe (median, IQR) & IMPROVE (mean, SD) \\
\hline \multicolumn{4}{|c|}{ Time waiting for procedure } \\
\hline EVAR & $74 \min (39-126)$ & & $93 \min ( \pm 370)$ \\
\hline Open repair & $45 \min (35-70)$ & & $73 \min ( \pm 157)$ \\
\hline \multicolumn{4}{|c|}{ Time in operating theatre } \\
\hline EVAR & $185 \min (160-236)$ & $160 \min (150-234)$ & $156 \min ( \pm 100)$ \\
\hline Open repair & $157 \min (136-194)$ & $150 \min (141-204)$ & $180 \min ( \pm 107)$ \\
\hline \multicolumn{4}{|c|}{ Blood loss during operation } \\
\hline EVAR & $500 \mathrm{~mL}(200-1375)$ & 200 mL (163-450) & \\
\hline Open repair & $3500 \mathrm{~mL}(1000-4600)$ & $2100 \mathrm{~mL}(1150-3985)$ & \\
\hline \multicolumn{4}{|c|}{ Length of hospital stay } \\
\hline EVAR & 9 days $(4-21)$ & 10 days $(6-28)$ & 9.8 days $( \pm 9.0)$ \\
\hline Open repair & 13 days $(5-21)$ & 12 days (4-52) & 12.2 days $( \pm 10.2)$ \\
\hline
\end{tabular}


This is the first systematic review, evaluating only studies that are prospective, RCTs, comparing eEVAR with open repair in patients with RAAA. There have been other studies, including several systematic reviews, that have addressed eEVAR versus open repair in patients with RAAA, but these have been mostly observational, non-randomised studies, many of which were retrospective. These types of studies are more likely subject to bias, compared with RCTs.

Visser et $a \bar{l}^{\tilde{6}}$ included 10 observational studies comparing patients who underwent eEVAR or open surgery, looking at short-term outcomes. A crude random-effects model for 30-day mortality, comparing eEVAR with open repair found that when patient haemodynamic condition at presentation was included in the model, that both modalities are suitable for treatment of patients with RAAA, and that eEVAR may possibly have a higher 30-day survival. The crude and adjusted ORs showed a stronger relationship between lower mortality in the eEVAR group than our own results for the 30-day mortality outcome, which showed no difference between the two interventions. The Visser study also evaluated a composite, systemic complications outcome, which found a lower point estimate within the eEVAR group, $28 \%$ (95\% CI $17 \%$ to $48 \%$ ), compared with open repair, $56 \%$ (95\% CI $37 \%$ to $85 \%$ ), indicating fewer complications within the eEVAR group. ${ }^{56}$ The present study did not have sufficient data on complications to compare with these results, nor was a composite, systemic complications outcome included.

The Takagi and Umemoto ${ }^{57}$ meta-analysis included 11 RCTs or risk-adjusted observational studies, with a total of 42888 patients. Inclusion criteria required studies to be RCTs or risk-adjusted observational comparative studies. This review included 1 RCT and 10 observational studies. While the present mortality results showed little difference between eEVAR and open repair, the Takagi study showed a strong relationship of lower mortality in the eEVAR arm $(\mathrm{p}<0.0001) .{ }^{57}$

Another meta-analysis included 18 studies, of which 11 were retrospective, and 2 of the RCTs in the present review. ${ }^{58}$ The review demonstrated a lower mortality and shorter length of stay in the eEVAR group $(p=0.01)$. However, the heterogeneity of study designs in the meta-analysis significantly detracts from the quality of the results and conclusions.

Another review included 41 studies, of which 2 were RCTs, and the remaining were observational, population-based studies, with a total of 59941 participants. ${ }^{59}$ The authors found a statistically significant lower mortality for the participants who underwent eEVAR, compared with open repair, OR $0.56(95 \%$ CI 0.50 to $0.64 ; \mathrm{p}<0.00001)$. The mortality outcome showed a strong mortality odds reduction for the eEVAR group where our study found little difference. Many of the complications evaluated in the Antoniou study also showed lower risk in the eEVAR group. The authors also evaluated cardiac complications, showing a borderline statistically significant risk difference favouring eEVAR $(-0.02,95 \%$ CI -0.03 to $0.00 ; p=0.05)$. The findings of complication outcomes in our review cannot currently be compared as there is not enough data to make any definitive conclusions.

Owing to a lack of data, this present review, using only prospective RCTs, was unable to determine superiority of either eEVAR or open repair for RAAA. Previous retrospective studies have suggested a significant survival advantage in case-control study designs for patients undergoing eEVAR. However, the key difference between eEVAR and an open historical comparative group is the anatomical configuration. Favourable anatomy to EVAR may preselect patients who ultimately will do well despite the modality of surgery. So when only these selected patients are included in a RCT, no difference is found in outcomes. Based on these findings, it would be difficult to justify complex endovascular repair of juxtarenal or thoracoabdominal aneurysms in the emergency setting. Caution also needs to be voiced in transferring a patient with RAAA from a centre which can provide open repair, to an alternative centre equipped to provide both open and endovascular repair, with a consequent delay in treatment. In addition, while volume outcome analysis supports centralisation, the provision of both modes of RAAA repair does not.

Further trials to evaluate the role of eEVAR in the treatment of RAAA are required, addressing all outcome measures. The evidence from non-randomised studies, showing reduced mortality in selected patients deemed suitable for endovascular repair, may raise ethical concerns in relation to randomising these patients to open repair. It may, therefore, be necessary to stratify the patients according to anatomical criteria at the time of CT diagnosis, so that the studies are powered for subgroups analysis.

Acknowledgements The authors wish to thank the Cochrane PVD Group for their support in approving this publication of the recent Cochrane Review (CD005261). This article is based on a Cochrane Review published in the Cochrane Database of Systematic Reviews (CDSR) 2014, Issue 7, DOI: 10.1002/14651858.CD005261 (see http://www.thecochranelibrary.com for information). Cochrane Reviews are regularly updated as new evidence emerges and in response to feedback, and the CDSR should be consulted for the most recent version of the review.

Contributors This paper is an update of the original Cochrane review in 2007. $\mathrm{SAB}, \mathrm{DWH}, \mathrm{PHB}, \mathrm{PKE}, \mathrm{FK}$ and Bedenis $\mathrm{R}$ were involved in study design. $\mathrm{SAB}$ and Bedenis $R$ were involved in data collection and data analysis. SAB, DWH, $\mathrm{PHB}, \mathrm{PKE}, \mathrm{FK}$ and Bedenis $\mathrm{R}$ were involved in writing the manuscript and final approval of the manuscript.

Funding This research received no specific grant from any funding agency in the public, commercial or not-for-profit sectors.

Competing interests None declared.

Provenance and peer review Not commissioned; externally peer reviewed.

Data sharing statement No additional data are available.

Open Access This is an Open Access article distributed in accordance with the Creative Commons Attribution Non Commercial (CC BY-NC 4.0) license, 
which permits others to distribute, remix, adapt, build upon this work noncommercially, and license their derivative works on different terms, provided the original work is properly cited and the use is non-commercial. See: http:// creativecommons.org/licenses/by-nc/4.0/

\section{REFERENCES}

1. Conway AM, Malkawi $\mathrm{AH}$, Hinchliffe $\mathrm{RJ}$, et al. First-year results of a national abdominal aortic aneurysm screening programme in a single centre. Br J Surg 2012;99:73-7.

2. Anon. NHS Abdominal Aortic Aneurysm Screening Programme. 2013. http://aaa.screening.nhs.uk

3. Alsac JM, Kobeiter $\mathrm{H}$, Becquemin JP et al. Endovascular repair for ruptured AAA: a literature review. Acta Chir Belg 2005;105:134-9.

4. Korhonen SJ, Ylonen K, Biancari F, et al, Finnvasc Study Group. Glasgow Aneurysm Score as a predictor of immediate outcome after surgery for ruptured abdominal aortic aneurysm. Br J Surg 2004;91:1449-52.

5. Neary WD, Crow P, Foy C, et al. Comparison of POSSUM scoring and the Hardman Index in selection of patients for repair of ruptured abdominal aortic aneurysm. Br J Surg 2003;90:421-5.

6. Hinterseher I, Saeger HD, Koch R, et al. Quality of life and long-term results after ruptured abdominal aortic aneurysm. Eur $J$ Vasc Endovasc Surg 2004;28:262-9.

7. Tambyraja AL, Fraser SC, Murie JA, et al. Quality of life after repair of ruptured abdominal aortic aneurysm. Eur J Vasc Endovasc Surg 2004;28:229-33.

8. Gorham TJ, Taylor J, Raptis S. Endovascular treatment of abdominal aortic aneurysm. Br J Surg 2004;91:815-27.

9. Greenhalgh RM, Forbes JF, Fowkes FG, et al. Early elective open surgical repair of small abdominal aortic aneurysms is not recommended: results of the UK Small Aneurysm Trial. Steering Committee. Eur J Vasc Endovasc Surg 1998;16:462-4.

10. Veith FJ, Ohki T, Lipsitz EC, et al. Treatment of ruptured abdominal aortic aneurysms with stent grafts: a new gold standard? Semin Vasc Surg 2003;16:171-5.

11. Ashton HA, Buxton MJ, Day NE, et al. Multicentre Aneurysm Screening Study Group. The Multicentre Aneurysm Screening Study (MASS) into the effect of abdominal aortic aneurysm screening on mortality in men: a randomised controlled trial. Lancet 2002;360:1531-9.

12. Scott RA, Ashton HA, Kay DN. Abdominal aortic aneurysm in 4237 screened patients: prevalence, development and management over 6 years. Br J Surg 1991;78:1122-5.

13. Harris $\mathrm{P}$, Buth $\mathrm{J}$, Eurostar, et al. What is the future for registries on endovascular aneurysm repair and who should be responsible? Eur J Vasc Endovasc Surg 2005;30:343-5

14. Thomas SM, Beard JD, Ireland M, et al, Vascular Society of Great Britain and Ireland; British Society of Interventional Radiology. Results from the prospective registry of endovascular treatment of abdominal aortic aneurysms (RETA): mid-term results to five years. Eur J Vasc Endovasc Surg 2005;29:563-70.

15. Lee WA, Herniese CM, Tayyarah M, et al. Impact of endovascular repair on early outcomes of ruptured abdominal aortic aneurysms. J Vasc Surg 2004;40:211-15.

16. Greenhalgh RM, Brown LC, Kwong GP, et al, EVAR trial participants. Comparison of Endovascular aneurysm repair with open repair in patients with abdominal aortic aneurysm (EVAR tria 1), 30-day operative mortality results: randomised controlled trial. Lancet 2004;364:843-8.

17. Prinssen M, Verhoeven ELG, Buth J, et al. A randomized controlled trial comparing conventional and endovascular repair of abdominal aortic aneurysms. N Engl J Med 2004;351:1607-18.

18. Lachat ML, Pfammatter T, Wizke HJ, et al. Endovascular repair with bifurcated stent-grafts under local anaesthesia to improve outcome of ruptured aortoiliac aneurysms. Eur J Vasc Endovasc Surg 2002;23:528-36.

19. EVAR trial participants. Endovascular aneurysm repair versus open repair in patients with abdominal aortic aneurysm (EVAR trial 1): randomised controlled trial. Lancet 2005;365:2179-86.

20. EVAR trial participants. Endovascular aneurysm repair and outcome in patients unfit for open repair of abdominal aortic aneurysm (EVAR trial 2): randomised controlled trial. Lancet 2005;365: 2187-92.

21. Brown LC, Powell JT, Thompson SG, et al. The UK Endovascular Aneurysm Repair (EVAR) trials: randomised trials of EVAR versus standard therapy. Health Technol Assess 2012;16:1-218.

22. Adam DJ, Mohan IV, Stuart WP, et al. Community and hospital outcome from ruptured abdominal aortic aneurysm within the catchment area of a regional vascular surgical service. $J$ Vasc Surg 1999;30:922-8

23. Huber TS, Harward TR, Flynn TC, et al. Operative mortality rates after elective infrarenal aortic reconstructions. J Vasc Surg 1995;22:287-93; discussion 293-4.

24. Lindsay TF, Luo XP, Lehotay DC, et al. Ruptured abdominal aortic aneurysm, a "two-hit" ischaemia/reperfusion injury: evidence from an analysis of oxidative products. J Vasc Surg 1999;30:219-28.

25. Bown MJ, Nicholson ML, Bell PR, et al. The systemic inflammatory response syndrome, organ failure and mortality after abdominal aortic aneurysm repair. J Vasc Surg 2003;37:600-6.

26. Harris LM, Faggioli GL, Fiedler R, et al. Ruptured abdominal aortic aneurysms: factors affecting mortality rates. J Vasc Surg 1991;14:812-18; discussion 819-20.

27. Roumen RM, Hendriks T, van der Ven-Jongekrijg J, et al. Cytokine patterns in patients after major vascular surgery, haemorrhagic shock and severe blunt trauma. Relation with subsequent adult respiratory distress syndrome and multiple organ failure. Ann Surg 1993;218:769-76

28. Cuypers PWM, Gardien M, Buth J, et al. Randomized study comparing cardiac response in endovascular and open aortic aneurysm repair. Br J Surg 2001;88:1059-65.

29. Peppelenbosch N, Yilmaz N, van Marrewijk C, et al. Emergency treatment of acute symptomatic or ruptured abdominal aortic aneurysm. Outcome of a prospective intent-to-treat by EVAR protocol. Eur J Vasc Endovasc Surg 2003;26:303-10.

30. van Sambeek MR, van Dijk LC, Hendriks JM, et al. Endovascular versus conventional open repair of acute abdominal aortic aneurysm: feasibility and preliminary results. J Endovasc Ther 2002;9:443-8

31. Makar RR, Badger SA, O'Donnell ME, et al. The inflammatory response to ruptured abdominal aortic aneurysm is altered by endovascular repair. Intern J Vasc Med 2013;2013:482728.

32. Makar RR, Badger SA, O'Donnell ME, et al. The impact of endovascular repair of ruptured abdominal aortic aneurysm on gastrointestinal and renal function. Intern J Vasc Med 2014 178323. doi:10.1155/2014/178323

33. Badger S, Bedenis R, Blair $\mathrm{PH}$, et al. Endovascular treatment of ruptured abdominal aortic aneurysm. Cochrane Database Syst Rev 2014;7:CD005261.

34. Dillon M, Cardwell C, Blair PH, et al. Endovascular treatment of ruptured abdominal aortic aneurysm. Cochrane Database Syst Rev 2007;(1):CD005261

35. The Cochrane library. http:www.thecochranelibrary.com

36. Higgins JPT, Altman DG, Sterne JAC, eds. Cochrane Handbook for Systematic Review of Interventions Version 5.1.0 (updated March 2011). Chapter 8: assessing risk of bias in included studies. http:// www.cochranehandbook.org

37. Higgins JP, Thompson SG, Deeks JT, et al. Measuring inconsistency in meta-analyses. BJM 2003;327:557-60.

38. Hinchliffe RJ, Bruijstens L, MacSweeney STR, et al. A randomised trial of endovascular and open surgery for ruptured abdominal aortic aneurysm-results of a pilot study and lessons learned for future studies. Eur J Vasc Endovasc Surg 2006;32:506-13; discussion 514-15.

39. Anon. Acute endovascular treatment to improve outcome of ruptured aorto-iliac aneurysms. The AJAX trial. 2009. http://controlled-trials. $\mathrm{com} / \mathrm{mrct} /$ trial/229677/ajax

40. Balm R. Acute endovascular treatment to improve outcome of ruptured aortoiliac aneurysms. 2005. http://www.controlled-trials. com/ISRCTN66212637/ISRCTN66212637

41. Balm R. 2012. http://www.cxvascular.com/cx-latest-news/cx-latestnews/ajax-confirms-no-difference-between-evar-and-open-repair-forruptured-aneurysms

42. Hoornweg LL, Balm R, Legemate DA, et al. Amsterdam Acute Aneurysm Trial: background, design, and methods. Vascular 2006;14:130-5.

43. Hoornweg LL, Wisselink W, Vahl A, et al. Amsterdam-Acute-Aneurysm-Trial-Collaborators. The Amsterdam Acute Aneurysm Trial: suitability and application rate for endovascular repair of ruptured abdominal aortic aneurysms. Eur $J$ Vasc Endovasc Surg 2007;33:679-83.

44. Reimerink JJ, Hoornweg LL, Vahl AC, et al. Endovascular repair versus open repair of ruptured abdominal aortic aneurysms: a multicenter randomized controlled trial. Ann Surg 2013;258:248-56.

45. Powell JT, Thompson SG, Thompson MM, et al. The Immediate Management of the Patient with Rupture: Open Versus Endovascular repair (IMPROVE) aneurysm trial-ISRCTN 48334791 IMPROVE trialists. Acta Chir Belg 2009;109:678-80.

46. Powell JT, Hinchliffe RJ, Thompson MM, et al. Improve tria investigators. Observations from the IMPROVE trial concerning the 
clinical care of patients with ruptured abdominal aortic aneurysm Br J Surg 2014;101:216-24; discussion 224.

47. National Institute for Health Research. IMPROVE: can eEVAR reduce mortality from ruptured $A A A$ ? Protocol. National Health Institute for Health Research: Evaluation, Trials and Studies Coordinating Centre. http://www.hta.ac.uk/protocols/200700370064.pdf

48. Powell JT, Sweeting MJ, Thompson MM, et al. Endovascular or open repair strategy for ruptured abdominal aortic aneurysm: 30 day outcomes from IMPROVE randomised trial. BMJ 2014;348:f7661.

49. Rodel SG, Meerwaldt R, Beuk RJ, et al. Endovascular treatment of ruptured abdominal aortic aneurysm: is there a long-term benefit at follow-up? J Cardiovasc Surg 2012;53:83-9.

50. Visser JJ, Bosch JL, Hunink MG, et al. Endovascular repair versus open surgery in patients with ruptured abdominal aortic aneurysms: clinical outcomes with 1-year follow-up. J Vasc Surg 2006;44:1148-55.

51. Anon. Ruptured aorta-iliac aneurysms: endo vs surgery (ECAR). Clinicaltrials.gov 2007; NCT00577616. http://clinicaltrials.gov/ct2/ show/NCT00577616?term $=$ ECAR\&rank $=1$

52. Desgranges P. Ruptured aorta-iliac aneurysms: endo vs. surgery (ECAR). 2008. http://clinicaltrials.gov/ct2/show/NCT00577616? term $=$ stable+and+aortic\&rank $=2$
53. Desgranges $\mathrm{P}, \mathrm{Kobeiter} \mathrm{H}$, Castier $\mathrm{Y}$, et al. The Endovasculaire vs Chirurgie dans les Anevrysmes Rompus PROTOCOL trial update. $J$ Vasc Surg 2010;51:267-70.

54. Resch T, Malina M, Lindblad B, et al. Endovascular repair of ruptured abdominal aortic aneurysms: logistics and short-term results. J Endovasc Ther 2003;10:440-6.

55. Verhoeven EL, Prins TR, van den Dungen JJ, et al. Endovascular repair of acute AAAs under local anaesthesia with bifurcated endografts: a feasibility study. J Endovasc Ther 2002;9:729-35.

56. Visser JJ, van Sambeek MRHM, Hamza TH, et al. Ruptured abdominal aortic aneurysms: endovascular repair versus open surgery-systematic review. Radiology 2007;245:122-9.

57. Takagi $\mathrm{H}$, Umemoto $\mathrm{T}$. A meta-analysis of randomized and risk-adjusted observational studies of endovascular versus open repair for ruptured abdominal aortic aneurysm. Vasc Endovasc Surg 2012;45:717-19.

58. Qin C, Chen L, Xiao Y-B. Emergent endovascular versus open surgery repair for ruptured abdominal aortic aneurysms: a meta-analysis. PLOS ONE 2014;9:e87465.

59. Antoniou GA, Georgiadis GS, Antoniou SA, et al. Endovascular repair for ruptured abdominal aortic aneurysm confers an early survival benefit over open repair. J Vasc Surg 2013;58:1091-105. 